\title{
Problematic clinical isolates of Pseudomonas aeruginosa from the university hospitals in Sofia, Bulgaria: current status of antimicrobial resistance and prevailing resistance mechanisms
}

\begin{abstract}
Correspondence
Tanya Strateva

dr.strateva@abv.bg
\end{abstract}

Received 4 October 2006

Accepted 13 March 2007
Tanya Strateva, ${ }^{1}$ Vessela Ouzounova-Raykova, ${ }^{1}$ Boyka Markova, ${ }^{2}$ Albena Todorova, ${ }^{3}$ Yulia Marteva-Proevska ${ }^{2}$ and Ivan Mitov ${ }^{1}$

\author{
${ }^{1}$ Department of Microbiology, Medical University of Sofia, 2 Zdrave Street, 1431 Sofia, Bulgaria \\ ${ }^{2}$ Laboratory of Clinical Microbiology, Alexander University Hospital, Medical University of Sofia, \\ 1 Georgi Sofiiski Blvd, 1431 Sofia, Bulgaria \\ ${ }^{3}$ Laboratory of Molecular Pathology, University Hospital of Obstetrics and Gynecology, \\ Medical University of Sofia, 2 Zdrave Street, 1431 Sofia, Bulgaria
}

\begin{abstract}
A total of 203 clinical isolates of Pseudomonas aeruginosa was collected during 2001-2006 from five university hospitals in Sofia, Bulgaria, to assess the current levels of antimicrobial susceptibility and to evaluate resistance mechanisms to antipseudomonal antimicrobial agents. The antibiotic resistance rates against the following antimicrobials were: carbenicillin $93.1 \%$, azlocillin $91.6 \%$, piperacillin $86.2 \%$, piperacillin/tazobactam $56.8 \%$, ceftazidime $45.8 \%$, cefepime $48.9 \%$, cefpirome $58.2 \%$, aztreonam $49.8 \%$, imipenem $42.3 \%$, meropenem $45.5 \%$, amikacin $59.1 \%$, gentamicin $79.7 \%$, tobramycin $89.6 \%$, netilmicin $69.6 \%$ and ciprofloxacin $80.3 \%$. A total of 101 of the studied $P$. aeruginosa isolates $(49.8 \%)$ were multidrug resistant. Structural genes encoding class $A$ and class $D \beta$-lactamases showed the following frequencies: bla $a_{\mathrm{VEB}-1} 33.1 \%$, bla PSE-1 $_{2} 22.5 \%$, bla $a_{\mathrm{PER}-1} 0 \%$, bla $\mathrm{OXX}_{\text {-groupl }} 41.3 \%$ and bla $\mathrm{OXA-groupll} 8.8 \%$. IMP- and VIM-type carbapenemases were not detected. In conclusion, the studied clinical strains of $P$. aeruginosa were problematic nosocomial pathogens. VEB-1 extended-spectrum $\beta$-lactamases appear to have a significant presence among clinical $P$. aeruginosa isolates from Sofia. Carbapenem resistance was related to non-enzymic mechanisms such as a deficiency of OprD proteins and active efflux.
\end{abstract}

\section{INTRODUCTION}

Pseudomonas aeruginosa is responsible for $10-15 \%$ of nosocomial infections worldwide (Blanc et al., 1998). The infections are frequently difficult to treat because of both the natural resistance of the species and its remarkable ability to acquire further resistance mechanisms to multiple groups of antimicrobial agents. P. aeruginosa represents a phenomenon of antibiotic resistance, demonstrating practically all known enzymic and mutational mechanisms of bacterial resistance. These mechanisms are often present

\footnotetext{
Abbreviations: AAC, aminoglycoside acetyltransferase; ANT, aminoglycoside nucleotidyltransferase; ESBL, extended-spectrum $\beta$-lactamase; ICU, intensive care unit; LRTI, lower respiratory tract infection; MBL, metallo- $\beta$-lactamase; URTI, upper respiratory tract infection.

The GenBank/EMBL/DDBJ accession nos for the $P$. aeruginosa bla VEB-1 $_{1}$ and bla $\mathrm{PSE}-1$ gene sequences are D0333895 and M69058, respectively.
}

simultaneously, conferring combined resistance to many strains (McGowan, 2006).

Multidrug-resistant strains of $P$. aeruginosa (resistant to at least three of the following antimicrobials: ceftazidime, imipenem, gentamicin and ciprofloxacin) are often isolated among patients suffering from nosocomial infections, particularly those receiving intensive care treatment (Tassios et al., 1997). The increasing rate of $P$. aeruginosa strains in a wide spectrum of clinical settings determines them as emerging pathogens, especially in intensive care units (ICUs), and justifies the necessity for antimicrobialresistance surveillance.

The aim of this study was to assess the current levels of antimicrobial susceptibility and to evaluate the resistance mechanisms to antipseudomonal antimicrobial agents among problematic clinical isolates of $P$. aeruginosa collected from five university hospitals in Sofia, Bulgaria. 


\section{METHODS}

Bacterial isolates. A collection of 203 non-duplicate, problematic clinical isolates of $P$. aeruginosa (resistant to one or more of the following groups of antimicrobials: third- or fourth-generation cephalosporins, carbapenems, aminoglycosides and fluoroquinolones) were used in the present study. The strains were collected during the period 2001-2006 from in-patients of different types of ward in five university hospitals in Sofia: surgical, orthopaedic, internal, paediatric, neurological and ICUs. The isolates were obtained from urine (79), tracheal aspirates (27), sputum (14), bronchial lavage (12), pleural fluid (2), surgical wounds or abscesses (30), drainages (9), blood (9), nose (9), throat (7), ear (1), rectal swabs (2) and bile (2). Bacterial identification was performed using a BBL Enteric/Nonfermenter ID system (Becton Dickinson).

Antimicrobial-susceptibility testing. The susceptibility of the investigated $P$. aeruginosa isolates to 17 antimicrobial agents was determined by the disc diffusion method on Mueller-Hinton II agar plates (Becton Dickinson) using antibiotic-containing discs provided by Becton Dickinson, Mast Diagnostics and Bul Bio, and was interpreted according to the National Committee for Clinical Laboratory Standards (NCCLS) (now the Clinical and Laboratory Standards Institute) 2004 recommendations (NCCLS, 2004). Control strains included P. aeruginosa ATCC 27853 and Escherichia coli ATCC 25922.

\section{Phenotypic methods for detection of resistance mechanisms to antimicrobial agents}

Detection of group 1 inducible $\boldsymbol{\beta}$-lactamases. The prevalence of inducible AmpC $\beta$-lactamase (molecular class $\mathrm{C}$, functional group 1) (Bush et al., 1995) in the studied strains of $P$. aeruginosa was investigated using a disc approximation test method (Sanders \& Sanders, 1992). A ceftazidime $(30 \mu \mathrm{g})$ disc was placed at a distance of $20 \mathrm{~mm}$ (centre to centre) from an imipenem $(10 \mu \mathrm{g})$ disc on a Mueller-Hinton II agar plate inoculated with a suspension of the test organism, adjusted to a McFarland no. 0.5 tube. After overnight incubation, distinct flattening of the inhibitory zone around the ceftazidime-containing disc on the side nearest to the imipenem disc was taken to indicate the presence of inducible AmpC $\beta$-lactamase.
Screening for extended-spectrum $\boldsymbol{\beta}$-lactamases (ESBLs). The presence of ESBLs was investigated by the double disc synergy test (Jarlier et al., 1988). Ceftazidime $(30 \mu \mathrm{g})$, cefepime $(30 \mu \mathrm{g})$, cefpirome $(30 \mu \mathrm{g})$ and aztreonam $(30 \mu \mathrm{g})$ discs were placed next to an amoxicillin/clavulanic acid $(20 / 10 \mu \mathrm{g})$-containing disc at a distance of $20 \mathrm{~mm}$ (centre to centre) on a Mueller-Hinton II agar plate inoculated with the test organism. After overnight incubation at $37^{\circ} \mathrm{C}$, an enhancement of the inhibition zone around at least one of these discs toward the clavulanate-containing disc indicated the presence of ESBLs. All studied strains were tested additionally by a disc diffusion method with imipenem $(10 \mu \mathrm{g})$ and ceftazidime $(30 \mu \mathrm{g})$ discs for the presence of synergism (Weldhagen et al., 2003).

Screening for metallo- $\beta$-lactamases (MBLs). The presence of Ambler class B MBLs (Bush et al., 1995) was studied using the modified Hodge test (Lee et al., 2001).

Detection of presumptive aminoglycoside-modifying enzymes. This test was performed according to the substrate profile, as described by the Aminoglycoside Resistance Study Groups (1994).

PCR amplification and sequencing of $\boldsymbol{\beta}$-lactamase genes. Total DNA from $P$. aeruginosa isolates was extracted by boiling. The detection of $b l a_{\mathrm{VEB}-1}, b l a_{\mathrm{PER}-1}, b l a_{\mathrm{PSE}-1}, b l a_{\mathrm{OXA}-\text { groupI }}, b l a_{\mathrm{OXA}-\text { groupII }}$, $b l a_{\mathrm{IMP}-l i k e}$ and $b l a_{\mathrm{VIM}-\text { like }}$ genes in the investigated strains was performed by PCR with the specific primers (Alpha DNA) listed in Table 1. PCR was carried out with $2 \mu \mathrm{l}$ template DNA, $0.25 \mu \mathrm{M}$ each primer, $0.2 \mathrm{mM}$ deoxyribonucleoside triphosphates, $1 \times$ reaction buffer, $2 \mathrm{mM} \mathrm{MgCl}_{2}$ and $1.5 \mathrm{U}$ Prime Taq DNA polymerase (GENET $\mathrm{BIO})$ in a total volume of $25 \mu \mathrm{l}$. The DNA was amplified in a Techgen PCR thermocycler (Techne) using the following protocol: initial denaturation $\left(94^{\circ} \mathrm{C}\right.$ for $5 \mathrm{~min}$ ), followed by 30 cycles of denaturation $\left(94{ }^{\circ} \mathrm{C}\right.$ for $\left.45 \mathrm{~s}\right)$, annealing $\left(50-64{ }^{\circ} \mathrm{C}\right.$, from $45 \mathrm{~s}$ to $\left.1 \mathrm{~min}\right)$ and extension $\left(72^{\circ} \mathrm{C}\right.$, from $45 \mathrm{~s}$ to $\left.1 \mathrm{~min}\right)$, with a single final extension of 7 min at $72{ }^{\circ} \mathrm{C}$. PCR products were separated in $1 \%$ agarose gel for $50 \mathrm{~min}$ at $150 \mathrm{~V}$, stained with ethidium bromide $\left(0.5 \mu \mathrm{g} \mathrm{ml}^{-1}\right)$ and detected by a UV transillumination (wavelength $312 \mathrm{~nm}$ ). The amplified genes were identified on the basis of fragment size (shown in Table 1). Selected VEB-1 and PSE-1 PCR products were purified with ExoSAP-IT reagent (Amersham Biosciences). Sequencing

Table 1. Oligonucleotides used as primers for amplification and sequencing

\begin{tabular}{|c|c|c|c|c|c|}
\hline Primer $^{*}$ & Target & Sequence $\left(5^{\prime} \rightarrow 3^{\prime}\right) \dagger$ & $\begin{array}{l}\text { Product } \\
\text { size }(b p)\end{array}$ & $\begin{array}{c}\text { Annealing } \\
\text { temperature }\left({ }^{\circ} \mathrm{C}\right)\end{array}$ & Reference \\
\hline VEB-F & VEB-1 ESBL & CGACTTCCATTTCCCGATGC & 643 & 58 & Naas et al. (2000) \\
\hline PER-F & PER-1 ESBL & AATTTGGGCTTAGGGCAGAA & 925 & 50 & Claeys et al. (2000) \\
\hline PER-B & & ATGAATGTCATTATAAAAGC & & & \\
\hline PSE-F & PSE-1 & AATGGCAATCAGCGCTTC & 699 & 54 & De Champs et al. (2002) \\
\hline OXA-10-F & OXA group I & TCTTTCGAGTACGGCATTAGC & 759 & 58 & Naas et al. (2000) \\
\hline OXA-10-B & & CCAATGATGCCCTCACTTTCC & & & \\
\hline OXA-2-F & OXA group II & GCCAAAGGCACGATAGTTGT & 701 & 64 & De Champs et al. (2002) \\
\hline OXA-2-B & & GCGTCCGAGTTGACTGCCGG & & & \\
\hline IMP-F & IMP-type MBLs & GAAGGYGTTTATGTTCATAC & 587 & 56 & Nordmann \& Poirel (2002) \\
\hline IMP-B & & GTAMGTTTCAAGAGTGATGC & & & \\
\hline
\end{tabular}

${ }^{*}$ F, Forward; B, backward.

$\dagger \mathrm{Y}=\mathrm{C}$ or $\mathrm{T} ; \mathrm{M}=\mathrm{A}$ or $\mathrm{C}$. 
reactions were performed using the same $b l a_{\mathrm{VEB}-1^{-}}$and $b l a_{\mathrm{PSE}-1^{-}}$ specific primers and a BigDye terminator v3.1 kit (Applera) in an automated sequencer (ABI 310 sequence genetic analyser; Applied Biosystems). The nucleotide and deduced amino acid sequences were analysed with software available from the National Center for Biotechnology Information (http://www.ncbi.nlm.nih.gov).

Statistical analysis. Student's $t$-test was used to assess differences in resistance rates. A $P$ value below 0.05 was considered to be statistically significant.

\section{RESULTS AND DISCUSSION}

The antimicrobial-resistance testing results are presented in Table 2. The established antimicrobial resistances, in increasing order, were to: polymyxin $\mathrm{B}<$ imipenem $<$ meropenem $<$ ceftazidime $<$ cefepime $<$ aztreonam $<$ piperacillin/tazobactam. Polymyxin $\mathrm{B}$ remained active against all isolates. The antimicrobial resistance to antibiotics of the investigated problematic strains of $P$. aeruginosa was higher than the mean $P$. aeruginosa resistance found in Bulgaria in 2003, according to data from the national program BulSTAR: 45.8 vs $24.5 \%$ to ceftazidime, 42.3 vs $8.3 \%$ to imipenem, 59.1 vs $24.9 \%$ to amikacin, 79.7 vs $38.7 \%$ to gentamicin and 80.3 vs $30.7 \%$ to ciprofloxacin (Petrov et al., 2005). Approximately half of our isolates $(49.8 \%)$ were multidrug resistant.

The comparative temporary resistance rates among the studied $P$. aeruginosa isolates are shown in Fig. $1(\mathrm{a}, \mathrm{b})$. The strains isolated during 2004-2006 were significantly more resistant $(P<0.001)$ than those obtained during 2001-2003 to the following antibiotics: ceftazidime (79.5 vs $20.0 \%$ ), cefepime ( 75.0 vs $26.9 \%$ ), cefpirome (81.6 vs $7.6 \%$ ), aztreonam ( 73.9 vs $31.0 \%$ ), imipenem ( 59.1 vs $31.3 \%$ ), meropenem (62.5 vs $27.8 \%$ ) and amikacin (72.7 vs $47.8 \%$ ). Thus, these results demonstrated increasing resistance rates to extended-spectrum antipseudomonal cephalosporins, carbapenems, monobactams and amikacin in $P$. aeruginosa isolates from the university hospitals in Sofia during the last 3 years.

The strains of $P$. aeruginosa from ICUs were more resistant to antibiotics than the overall studied strains, except to cefpirome and aztreonam (Table 2). The ICU isolates were significantly more resistant to meropenem $(61.4 \%)$ than all investigated isolates as a whole $(45.5 \%, P<0.05)$, which is related to the widespread use of meropenem for the treatment of life-threatening infections in ICUs.

The antimicrobial resistance in $P$. aeruginosa varied among different clinical specimens (Table 2). The $P$. aeruginosa isolates from in-patients with lower respiratory tract infections (LRTIs) were more resistant to piperacillin than the isolates obtained from wounds and drainages $(P<0.05)$. The strains of $P$. aeruginosa isolated from inpatients with LRTIs and upper respiratory tract infections (URTIs) were more resistant to piperacillin/tazobactam than those from urine and wounds $(P<0.01$ and $P<0.001$, respectively). The observed resistance rate to ceftazidime in $P$. aeruginosa from wounds and drainages was lower than that in URTI isolates $(P<0.05)$. URTI strains of $P$. aeruginosa were more resistant to cefpirome than LRTI isolates $(P<0.05)$. The antibiotic resistance of $P$. aeruginosa

Table 2. Antimicrobial resistance as percentage of isolates from different sources among 203 isolates of $P$. aeruginosa

\begin{tabular}{|c|c|c|c|c|c|c|c|}
\hline $\begin{array}{l}\text { Antimicrobial } \\
\text { agent }\end{array}$ & Urine $(n=79)$ & LRTI $(n=55)$ & $\begin{array}{c}\text { Wounds and } \\
\text { drainages }(n=39)\end{array}$ & URTI $(n=17)$ & $\begin{array}{l}\text { Blood culture } \\
\qquad(n=9)\end{array}$ & ICU $(n=68)$ & $\begin{array}{c}\text { Total }^{\star} \\
(n=203)\end{array}$ \\
\hline Carbenicillin & 89.9 & 96.4 & 94.9 & 2.3 & 100.0 & 97.2 & 93.1 \\
\hline Azlocillin & 89.3 & 96.2 & 91.7 & 87.5 & 87.5 & 95.7 & 91.6 \\
\hline Piperacillin & 84.1 & 97.5 & 77.3 & 84.6 & 75.0 & 94.5 & 86.2 \\
\hline $\begin{array}{c}\text { Piperacillin }+ \\
\text { tazobactam }\end{array}$ & 47.9 & 75.0 & 34.5 & 81.2 & 37.5 & 70.5 & 56.8 \\
\hline Ceftazidime & 46.8 & 40.0 & 35.9 & 64.7 & 77.8 & 49.3 & 45.8 \\
\hline Cefoperazone & 85.3 & 94.0 & 77.1 & 75.0 & 87.5 & 95.6 & 86.2 \\
\hline Cefepime & 45.6 & 51.0 & 47.4 & 52.9 & 66.7 & 53.1 & 48.9 \\
\hline Cefpirome & 55.3 & 51.8 & 59.5 & 76.5 & 77.8 & 55.7 & 58.2 \\
\hline Aztreonam & 51.9 & 41.5 & 41.0 & 64.7 & 77.8 & 43.5 & 49.8 \\
\hline Imipenem & 32.9 & 43.6 & 43.6 & 64.7 & 77.8 & 52.1 & 42.3 \\
\hline Meropenem & 31.6 & 53.7 & 43.6 & 64.7 & 77.8 & 61.4 & 45.5 \\
\hline Amikacin & 59.5 & 58.2 & 74.4 & 17.6 & 77.8 & 66.2 & 59.1 \\
\hline Gentamicin & 86.1 & 79.6 & 92.3 & 29.4 & 88.9 & 82.8 & 79.7 \\
\hline Tobramycin & 93.7 & 96.3 & 97.4 & 35.3 & 88.9 & 95.7 & 89.6 \\
\hline Netilmicin & 70.5 & 79.1 & 74.4 & 35.3 & 44.4 & 75.0 & 69.6 \\
\hline Ciprofloxacin & 92.4 & 78.2 & 87.2 & 29.4 & 66.7 & 80.3 & 80.3 \\
\hline Polymyxin B & 0.0 & 0.0 & 0.0 & 0.0 & 0.0 & 0.0 & 0.0 \\
\hline
\end{tabular}

${ }^{\star}$ Antimicrobial resistance of all strains of $P$. aeruginosa, including two rectal swab and two bile isolates. 
(a)

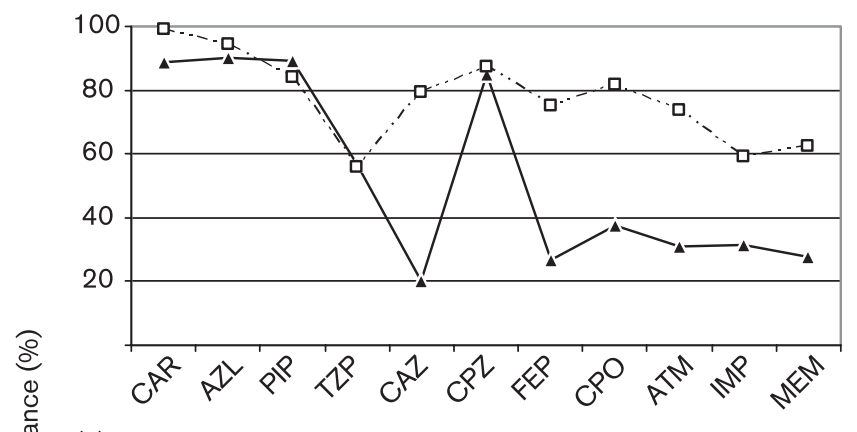

(b)

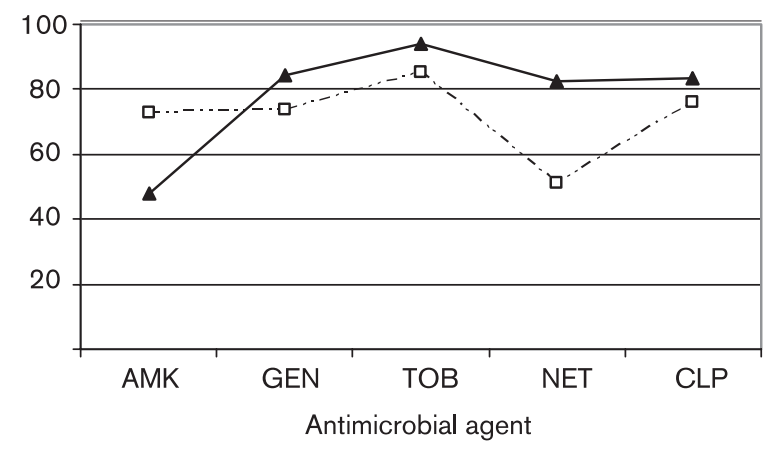

Fig. 1. Comparative temporary antimicrobial resistance in the studied $P$. aeruginosa isolates. $\boldsymbol{\Delta}$, 2001-2003 ( $n=115) ; \square$, 2004-2006 ( $n=88)$. (a) Resistance to $\beta$-lactams. CAR, Carbenicillin; AZL, azlocillin; PIP, piperacillin; TZP, piperacillin + tazobactam; CAZ, ceftazidime; CPZ, cefoperazone; FEP, cefepime; CPO, cefpirome; ATM, aztreonam; IMP, imipenem; MEM, meropenem. (b) Resistance to aminoglycosides and fluoroquinolones. AMK, Amikacin; GEN, gentamicin; TOB, tobramycin; NET, netilmicin; CIP, ciprofloxacin.

from urine samples towards imipenem and meropenem was lower than that among URTI strains $(P<0.02$ and $P<0.01$, respectively). URTI isolates were significantly more susceptible than the $P$. aeruginosa isolates from urine, LRTIs, and wounds and drainages to the following antimicrobials: amikacin $(P<0.001)$, gentamicin $(P<0.001)$, tobramycin $(P<0.001)$, netilmicin $(P<0.01$, $P<0.001$ and $P<0.01$, respectively) and ciprofloxacin $(P<0.001)$.

A total of 104 of the 203 investigated problematic $P$. aeruginosa isolates ( $51.2 \%)$ showed a 'penicillinase production phenotype' (resistance to carboxypenicillins and ureidopenicillins, and susceptibility to ceftazidime) (Bert et al., 2003). These strains were presumptive producers of narrow-spectrum $\beta$-lactamases. Additionally, they expressed an inducible AmpC $\beta$-lactamase (cephalosporinase).

The ceftazidime resistance rate was $45.8 \%$. A total of 57 of the strains of the 203 studied P. aeruginosa isolates (28.1\%) were resistant to extended-spectrum cephalosporins, including ceftazidime, and were characterized as presumptive producers of ESBLs according to the double disc synergy test. These strains also displayed in vitro synergism between imipenem and ceftazidime, typical of the producers of clavulanic acid- and tazobactam-inhibited ESBLs from molecular class A, such as VEB-, PER- or GES-type (Weldhagen et al., 2003).

Twelve strains of $P$. aeruginosa (5.9\%) were resistant to all $\beta$-lactams except carbapenems, and showed a negative result in the double disc synergy test. In these isolates, resistance to extended-spectrum cephalosporins was related mainly to the overproduction of a chromosomal AmpC cephalosporinase from molecular class C.

Of the 203 P. aeruginosa isolates, $12(5.9 \%)$ possessed a $\mathrm{OprD}^{-}$mutant phenotype (resistant only to imipenem and meropenem, but susceptible to other $\beta$-lactams). Carbapenem resistance is mostly due to $\mathrm{OprD}$ deficiency and is independent of susceptibility towards other $\beta$-lactam agents (Livermore, 2001). This resistance mechanism demands continued expression of the chromosomal AmpC $\beta$-lactamase (Livermore, 1992).

Overproduction of active efflux systems with wide substrate profiles was the prevailing resistance mechanism in eight $P$. aeruginosa isolates $(3.9 \%)$. In our study, the presumptive efflux systems were: MexA-MexB-OprM associated with decreased susceptibility or resistance to all $\beta$-lactams, except imipenem, and with decreased susceptibility or resistance to quinolones ( $\mathrm{nalB}$ or $\mathrm{nalC}$ mutants) (Llanes et al., 2004), and MexC-MexD-OprJ conferring resistance to fourth-generation cephalosporins (cefepime and cefpirome) and resulting from mutation in nfxB (Poole et al., 1996).

Sixty strains of $P$. aeruginosa (29.6\%) were resistant to all $\beta$-lactams, including carbapenems, and thus could be related to a phenotype of Ambler class B MBL-producing strains (Nordmann \& Poirel, 2002). All carbapenemresistant strains of $P$. aeruginosa showed a negative Hodge test and therefore were not producers of MBLs. Most probably, the resistance to $\beta$-lactams resulted from a combination of different mechanisms: OprD deficiency, derepression of chromosomal AmpC cephalosporinase, ESBL production and overexpression of active efflux systems.

One hundred and twenty isolates out of the studied strains of $P$. aeruginosa $(59.1 \%)$ were resistant to amikacin and 69.6-89.6\% to the other aminoglycosides (netilmicin, gentamicin and tobramycin). The most widespread mechanism of resistance to these antimicrobials involves enzymic modification by aminoglycoside acetyltransferases (AACs), aminoglycoside nucleotidyltransferases (ANTs) or aminoglycoside phosphotransferases (Poole, 2005). The prevailing phenotypes of aminoglycoside resistance in our strains were: (i) amikacin + gentamicin + tobramycin + netilmicin (49.9\%), associated with AAC (6')-I \pm ANT $\left(2^{\prime \prime}\right)$; (ii) gentamicin + tobramycin + netilmicin $(14.1 \%)$, associated with AAC (3)-V or ANT (2') + AAC (3)-Ia; and (iii) amikacin + gentamicin + tobramycin $(14.1 \%)$, related 
to aminoglycoside phosphotransferase (3')-VI + ANT (2' $)$ (Aminoglycoside Resistance Study Group, 1994).

One hundred and sixty three $(80.3 \%)$ of the isolates were resistant to ciprofloxacin. The most important mechanisms of quinolone resistance are structural alterations of the primary or secondary targets because of chromosomal point mutations in gyrA/gyrB or parC/parE genes, respectively, followed by an active efflux of these antimicrobial agents (Hooper, 2001).

A molecular genetic study was carried out for the presence of $\beta$-lactamases belonging to different molecular classes. A total of 160 isolates were investigated, and $53(33.1 \%)$ were found to be VEB-1 producers. The sequence of $b l a_{\mathrm{VEB}-1}$ amplified from different selected isolates was identical for all isolates and $100 \%$ identical to the known veb-1 sequence (GenBank accession no. DQ333895). The frequency of VEB-1 ESBLs among the ceftazidime-resistant $P$. aeruginosa was $57.0 \%$ (53/93). A total of 36 of the 160 isolates $(22.5 \%)$ produced PSE-1 enzyme. Selected PSE-1 PCR products showed $100 \%$ identity to $b l a_{\mathrm{PSE}-1}$ (GenBank accession no. M69058). The frequency of OXA group I and OXA group II $\beta$-lactamases was $41.3 \%(66 / 160)$ and $8.8 \%$ $(14 / 160)$, respectively.

The distribution of the Ambler class A and D $\beta$-lactamases among the investigated strains of $P$. aeruginosa is presented in Table 3. As shown, the relative proportion of $\beta$ lactamase-producing strains of $P$. aeruginosa $(66.8 \%)$ was higher than the proportion of $\beta$-lactamase-non-producing strains $(33.1 \%)$. An analogous study carried out recently in Korea established that $\beta$-lactamase-non-producing strains of $P$. aeruginosa were more widespread than producers (74.6 vs $25.4 \%$; Lee et al., 2005).

In our investigation, the $\beta$-lactamase producers were mostly present as VEB-1 + OXA group I (20.0\%), followed by PSE-1 (13.1\%) and OXA group I $(12.5 \%)$. The frequency of the Ambler class A $\beta$-lactamases (55.6\%) was approximately equal to the frequency of class $D$ $\beta$-lactamases $(50.1 \%)$. In comparison, class D OXA-type enzymes were detected more frequently than class A in $P$. aeruginosa from Korea (21.0 vs $6.3 \%$; Lee et al., 2005), in contrast to the data from Europe (31.3 vs $64.9 \%$; Bert et al., 2002).

A high frequency of distribution of ESBLs in the ceftazidime-resistant isolates of $P$. aeruginosa was established in our study. In all the university hospitals monitored in Sofia, widespread dissemination of $b l a_{\mathrm{VEB}-1}$ in clinical isolates of $P$. aeruginosa was found. Recently, Bachvarova et al. (2005) reported a significantly lower $(P<0.01)$ rate of prevalence of VEB-1-type $\beta$-lactamases among ceftazidime-resistant strains of $P$. aeruginosa obtained from distinct regions of Bulgaria during 19982003 than that determined in our study ( 36.8 vs $57.0 \%$ ). Thus, the observed trend towards an increasing rate of VEB-1-producing $P$. aeruginosa strains in Bulgaria relates to the last 2 years. VEB-1 and VEB-1-like enzymes are widespread in Asia (Thailand, Kuwait, India and China) (Weldhagen et al., 2003; Girlich et al., 2002; Poirel et al., 2001), but in European countries have been detected only in France (Naas et al., 1999).

A total of $42(26.3 \%)$ of the 160 isolates studied possessed both VEB-1 and OXA group I enzymes. It is likely that the strains produced the narrow-spectrum OXA-10 from OXA group I (Sanschagrin et al., 1995), encoded by a gene located on class 1 integron In50, as well as $b l_{\mathrm{VEB}-1}$ (Girlich et al., 2002). Lee et al. (2005) reported that OXA-10 was the most prevalent enzyme $(13.5 \%)$ in Korea in 2002.

PSE-1 $\beta$-lactamases belonging to Ambler class $\mathrm{A}$ and functional group 2c (Bush et al., 1995) were detected in $22.5 \%$ of the investigated strains. In 2002, Nordmann (2002) reported $11 \%$ CARB-producing strains of $P$. aeruginosa in France, $90 \%$ of which were PSE-1.

Our study did not reveal $b l a_{\mathrm{PER}-1}$, in contrast to the widespread detection of these genes in Europe. Epidemics caused by PER-1-producing $P$. aeruginosa have been reported previously in Turkey and Italy (Vahaboglu et al., 1997; Luzzaro et al., 2001). Isolates of $P$. aeruginosa with

Table 3. Prevalence of Ambler class $A$ and $D \beta$-lactamases in $160 P$. aeruginosa isolates

\begin{tabular}{|llc|}
\hline Class & Type of $\boldsymbol{\beta}$-lactamase & No. $(\%)$ of isolates \\
\hline Class A & PSE-1 & $21(13.1)$ \\
& VEB-1 & $8(5.0)$ \\
Class D & VEB-1 and PSE-1 & $2(1.3)$ \\
& OXA group II & $20(12.5)$ \\
Combined & OXA group I & $10(6.3)$ \\
& VEB-1 and OXA group I & $32(20.0)$ \\
& VEB-1, PSE-1 and OXA group I & $8(5.0)$ \\
& VEB-1, PSE-1, OXA group I and OXA group II & $2(1.3)$ \\
& PSE-1 and OXA group I & $2(1.3)$ \\
& PSE-1 and OXA group II & $1(0.6)$ \\
None & VEB-1, OXA group I and OXA group II & $1(0.6)$ \\
& $\beta$-lactamase-non-producing & $53(33.1)$ \\
\hline
\end{tabular}


PER-1 enzymes have also been observed in France, Belgium and Poland (De Champs et al., 2002; Claeys et al., 2000; Empel et al., 2005).

The established frequency of OXA group II $\beta$-lactamases comprising OXA-2, -3, -15 and -20 (Sanschagrin et al., 1995) was the lowest in our research. It is likely that the oxacillinases from group II were predominantly narrow spectrum, such as OXA-2 or -3 , as these enzymes were detected mainly in ceftazidime-susceptible strains of $P$. aeruginosa. In comparison, OXA group II enzymes were disseminated among $2.3 \%$ of the studied $P$. aeruginosa isolates in Korea and all strains were determined as OXA-2 producers (Lee et al., 2005). The frequency of bla $a_{\text {OXA-groupII }}$ in our strains of $P$. aeruginosa $(8.8 \%)$ was similar to the dissemination rate of OXA group II $\beta$-lactamases in France during 1994-1999 (9.9\%) (Bert et al., 2002).

Carbapenem-hydrolysing IMP- and VIM-type metalloenzymes belonging to Ambler class B were not detected in this study. The investigated carbapenem-resistant strains of $P$. aeruginosa from Sofia did not harbour $b l_{\mathrm{VIM}-\text { like }}$ genes, although the detection of these genes is widespread, especially in neighbouring countries such as Greece and Turkey (Tsakris et al., 2000; Mavroidi et al., 2000; Pournaras et al., 2002; Bahar et al., 2004). The carbapenem resistance was related to non-enzymic mechanisms such as OprD deficiency and active efflux.

The comparative antimicrobial resistances of $\beta$-lactamaseproducing and $\beta$-lactamase-non-producing $P$. aeruginosa are summarized in Table 4 . The $\beta$-lactamase producers were significantly more resistant than non-producers to ceftazidime, cefepime, cefpirome, aztreonam, amikacin, tobramycin and ciprofloxacin $(P<0.001)$ and to gentamicin
$(P<0.01)$. The susceptibilities to extended-spectrum cephalosporins, aztreonam and carbapenems among $\beta$ lactamase-producing strains of $P$. aeruginosa were lower than those in non- $\beta$-lactamase producers and were similar to the susceptibilities in analogous $P$. aeruginosa isolates from Korea in 2002 (Lee et al., 2005). Moreover, in our study and the Korean study, the cross-class resistance to aminoglycosides and ciprofloxacin was significantly higher in class A and D $\beta$-lactamase-producing $P$. aeruginosa. As described previously, VEB-1 was the first class A enzyme found to be encoded by an integron-located gene cassette (Poirel et al., $1999)$. In the $b l a_{\mathrm{VEB}-1}-$ containing integrons of $P$. aeruginosa, the veb-1 cassette is often associated with aminoglycoside resistance gene cassettes (Girlich et al., 2002).

In conclusion, the studied clinical strains of $P$. aeruginosa were problematic nosocomial pathogens and half were found to be multidrug resistant. From 2001 to 2006, the rates of resistance to third- or fourth-generation cephalosporins, monobactams, carbapenems and amikacin showed significant increases among the investigated $P$. aeruginosa strains from the monitored hospitals. The interpretation of the phenotypic patterns of antimicrobial susceptibility showed a variety of resistance mechanisms, from which the prevalent were expression of an inducible AmpC cephalosporinase, production of ESBLs and a combination of mechanisms conferring resistance to multiple groups of antimicrobials. The carbapenem resistance was not related to enzymic hydrolysis by Ambler class B MBLs. The clavulanic acid-inhibited VEB-1-type ESBLs appear to have a significant presence among $P$. aeruginosa isolates in the university hospitals in Sofia and cause serious impediments in antimicrobial treatment and difficulties in limiting their dissemination in Bulgaria.

Table 4. Comparison of antimicrobial resistance (\%) between the class $A$ and/or class $D \beta$ lactamase-producing and -non-producing $P$. aeruginosa isolates

\begin{tabular}{|lccl|}
\hline Antimicrobial agent & $\begin{array}{c}\boldsymbol{\beta} \text {-Lactamase producing } \\
(\boldsymbol{n}=\mathbf{1 0 7})\end{array}$ & $\begin{array}{c}\boldsymbol{\beta} \text {-Lactamase non-producing } \\
(\boldsymbol{n}=\mathbf{5 3})\end{array}$ & $\boldsymbol{P}$ value \\
\hline Carbenicillin & 100.0 & 98.1 & \\
Azlocillin & 99.1 & 98.1 & $P>0.1$ \\
Piperacillin & 90.5 & 90.9 & $P>0.1$ \\
Piperacillin t tazobactam & 48.4 & 60.5 & $P>0.1$ \\
Ceftazidime & 69.2 & 28.3 & $P>0.1$ \\
Cefoperazone & 95.2 & 90.2 & $P<0.001$ \\
Cefepime & 72.5 & 35.3 & $P>0.1$ \\
Cefpirome & 79.4 & 49.1 & $P<0.001$ \\
Aztreonam & 67.6 & 35.8 & $P<0.001$ \\
Imipenem & 53.3 & 41.5 & $P<0.001$ \\
Meropenem & 52.3 & 47.2 & $P>0.1$ \\
Amikacin & 77.6 & 35.8 & $P>0.1$ \\
Gentamicin & 88.8 & 67.9 & $P<0.001$ \\
Tobramycin & 99.1 & 75.5 & $P<0.01$ \\
Netilmicin & 65.0 & 73.1 & $P<0.001$ \\
Ciprofloxacin & 91.6 & 67.9 & $P>0.1$ \\
& & & $P<0.001$ \\
\hline
\end{tabular}




\section{ACKNOWLEDGEMENTS}

This work was supported by grant 05-2005 of the Medical University of Sofia, Bulgaria.

\section{REFERENCES}

Aminoglycoside Resistance Study Groups (1994). Resistance to aminoglycosides in Pseudomonas. Trends Microbiol 2, 347-353.

Bachvarova, A., Velinov, T., Petrov, M., Kantardjiev, T., Levterova, V. \& Ivanov, I. (2005). Widespread detection of VEB-1-type extendedspectrum $\beta$-lactamases among nosocomial Pseudomonas aeruginosa isolates in Bulgaria: a nationwide multicentre study. Clin Microbiol Infect 11 (Suppl. 2), 233.

Bahar, G., Mazzariol, A., Koncan, R., Mert, A., Fontana, R., Rossolini, G. M. \& Cornaglia, G. (2004). Detection of VIM-5 metallo- $\beta$ lactamase in a Pseudomonas aeruginosa clinical isolate from Turkey. $J$ Antimicrob Chemother 54, 282-283.

Bert, F., Branger, C. \& Lambert-Zechovsky, N. (2002). Identification of PSE and OXA $\beta$-lactamase genes in Pseudomonas aeruginosa using PCR-restriction fragment length polymorphism. I Antimicrob Chemother 50, 11-18.

Bert, F., Ould-Hocine, Z., Juvin, M., Dubois, V., Loncle-Provot, V., Lefranc, V., Quentin, C., Lambert, N. \& Arlet, G. (2003). Evaluation of the Osiris expert system for identification of $\beta$-lactam phenotypes in isolates of Pseudomonas aeruginosa. J Clin Microbiol 41, 3712-3718.

Blanc, D. S., Petignat, C., Janin, B., Bille, J. \& Francioli, P. (1998). Frequency and molecular diversity of Pseudomonas aeruginosa upon admission and during hospitalization: a prospective epidemiologic study. Clin Microbiol Infect 4, 242-247.

Bush, K., Jacoby, G. A. \& Medeiros, A. A. (1995). A functional classification scheme for $\beta$-lactamases and its correlation with molecular structure. Antimicrob Agents Chemother 39, 1211-1233.

Claeys, G., Verschraegen, G., de Baere, T. \& Vaneechoutte, M. (2000). PER-1 $\beta$-lactamase-producing Pseudomonas aeruginosa in an intensive care unit. J Antimicrob Chemother 45, 924-925.

De Champs, C., Poirel, L., Bonnet, R., Sirot, D., Chanal, C., Sirot, J. \& Nordmann, P. (2002). Prospective survey of $\beta$-lactamases produced by ceftazidime-resistant Pseudomonas aeruginosa isolated in a French hospital in 2000. Antimicrob Agents Chemother 46, 3031-3034.

Empel, J., Filczak, K., Mrowka, A., Hryniewicz, W. \& Gniadkowski, M. (2005). The first incidence of PER-1 ESBL-producing Pseudomonas aeruginosa in Poland and identification of a novel OXA $\beta$-lactamase variant. Clin Microbiol Infect 11 (Suppl. 2), 235.

Girlich, D., Naas, T., Leelaporn, A., Poirel, L., Fennewald, M. \& Nordmann, P. (2002). Nosocomial spread of the integron-located veb1 -like cassette encoding an extended-spectrum $\beta$-lactamase in Pseudomonas aeruginosa in Thailand. Clin Infect Dis 34, 603-611.

Hooper, D. C. (2001). Emerging mechanisms of fluoroquinolone resistance. Emerg Infect Dis 7, 337-341.

Jarlier, V., Nicolas, M. H., Fournier, G. \& Philippon, A. (1988). Extended broad-spectrum $\beta$-lactamases conferring transferable resistance to newer $\beta$-lactam agents in Enterobacteriaceae: hospital prevalence and susceptibility patterns. Rev Infect Dis 10, 867-878.

Lee, K., Chong, Y., Shin, H. B., Kim, Y. A., Yong, D. \& Yum, J. H. (2001). Modified Hodge and EDTA-disk synergy tests to screen metallo- $\beta$-lactamase-producing strains of Pseudomonas and Acinetobacter species. Clin Microbiol Infect 7, 88-91.

Lee, S., Park, Y. J., Kim, M., Lee, H. K., Han, K., Kang, C. S. \& Kang, M. W. (2005). Prevalence of Ambler class A and D $\beta$-lactamases among clinical isolates of Pseudomonas aeruginosa in Korea. J Antimicrob Chemother 56, 122-127.

Livermore, D. M. (1992). Interplay of impermeability and chromosomal $\beta$-lactamase activity in imipenem-resistant Pseudomonas aeruginosa. Antimicrob Agents Chemother 36, 2046-2048.

Livermore, D. M. (2001). Of Pseudomonas, porins, pumps and carbapenems. J Antimicrob Chemother 47, 247-250.

Llanes, C., Hocquet, D., Vogne, C., Benali-Baitich, D., Neuwirth, C. \& Plesiat, P. (2004). Clinical strains of Pseudomonas aeruginosa overproducing MexA-MexB-OprM and MexXY efflux pumps simultaneously. Antimicrob Agents Chemother 48, 1797-1802.

Luzzaro, F., Mantengoli, E., Perilli, M., Lombardi, G., Orlandi, V., Orsatti, A., Amicosante, G., Rossolini, G. M. \& Toniolo, A. (2001). Dynamics of a nosocomial outbreak of multidrug-resistant Pseudomonas aeruginosa producing the PER-1 extended-spectrum $\beta$-lactamase. J Clin Microbiol 39, 1865-1870.

Mavroidi, A., Tsakris, A., Tzelepi, E., Pournaras, S., Loukova, V. \& Tzouvelekis, L. S. (2000). Carbapenem-hydrolyzing VIM-2 metallo$\beta$-lactamase in Pseudomonas aeruginosa from Greece. J Antimicrob Chemother 46, 1041-1042.

McGowan, J. E. (2006). Resistance in nonfermenting Gram-negative bacteria: multidrug resistance to the maximum. Am J Med 119, S29-S36.

Naas, T., Poirel, L., Karim, A. \& Nordmann, P. (1999). Molecular characterization of In50, a class 1 integron encoding the gene for the extended-spectrum $\beta$-lactamase VEB-1 in Pseudomonas aeruginosa. FEMS Microbiol Lett 176, 411-419.

Naas, T., Benaoudia, F., Massuard, S. \& Nordmann, P. (2000). Integron-located VEB-1 extended-spectrum $\beta$-lactamase gene in a Proteus mirabilis clinical isolate from Vietnam. J Antimicrob Chemother 46, 703-711.

NCCLS (2004). Performance Standards for Antimicrobial Susceptibility Testing, 14th informational supplement, M100-S14. Wayne, PA: National Committee for Clinical Laboratory Standards.

Nordmann, P. (2002). Old and novel mechanisms of resistance to $\beta$ lactams in $P$. aeruginosa. In 7th ECC Symposium Anything new for $P$. aeruginosa?, 4-7 May 2002, Paris.

Nordmann, P. \& Poirel, L. (2002). Emerging carbapenemases in Gram-negative aerobes. Clin Microbiol Infect 8, 321-331.

Petrov, M., Hadjieva, N., Kantardjiev, T., Velinov, T. \& Bachvarova, A. (2005). Surveillance of antimicrobial resistance in Bulgaria - a synopsis from BulSTAR 2003. Euro Surveill 10, 79-82.

Poirel, L., Naas, T., Guibert, M., Chaibi, E. B., Labia, R. \& Nordmann, P. (1999). Molecular and biochemical characterization of VEB-1, a novel class A extended-spectrum $\beta$-lactamase encoded by an Escherichia coli integron gene. Antimicrob Agents Chemother 43, 573-581.

Poirel, L., Rotimi, V., Mokaddas, E., Karim, A. \& Nordmann, P. (2001). VEB-1-like extended-spectrum $\beta$-lactamases in Pseudomonas aeruginosa, Kuwait. Emerg Infect Dis 7, 468-470.

Poole, K. (2005). Aminoglycoside resistance in Pseudomonas aerginosa. Antimicrob Agents Chemother 49, 479-487.

Poole, K., Gotoh, N., Tsujimoto, H., Zhao, Q., Wada, A., Yamasaki, T., Neshat, S., Yamagishi, J., Li, X. Z. \& Nishino, T. (1996). Overexpression of the mexC-mexD-oprJ efflux operon in $n f x B$-type multidrug resistant strains of Pseudomonas aeruginosa. Mol Microbiol 21, 713-724.

Pournaras, S., Tsakris, A., Maniati, M., Tzouvelekis, L. S. \& Maniatis, A. N. (2002). Novel variant $\left(b l a_{\mathrm{VIM}-4}\right)$ of the metallo- $\beta$-lactamase gene $b l a_{\mathrm{VIM}-1}$ in a clinical strain of Pseudomonas aeruginosa. Antimicrob Agents Chemother 46, 4026-4028. 
Sanders, C. C. \& Sanders, W. E. (1992). $\beta$-Lactam resistance in Gram-negative bacteria: global trends and clinical impact. Clin Infect Dis 15, 824-839.

Sanschagrin, F., Couture, F. \& Levesque, R. C. (1995). Primary structure of OXA-3 and phylogeny of oxacillin-hydrolyzing class D $\beta$ lactamases. Antimicrob Agents Chemother 39, 887-893.

Tassios, P. T., Gennimata, V., Spaliara-Kalogeropoulou, L., Kairis, D., Koutsia, C., Vatopoulos, A. C. \& Legakis, N. J. (1997). Multiresistant Pseudomonas aeruginosa serogroup $\mathrm{O}: 11$ outbreak in an intensive care unit. Clin Microbiol Infect 3, 621-628.

Tsakris, A., Pournaras, S., Woodford, N., Palepou, M. F., Babini, G. S., Douboyas, J. \& Livermore, D. M. (2000). Outbreak of infections caused by Pseudomonas aeruginosa producing VIM-1 carbapenemase in Greece. J Clin Microbiol 38, 1290-1292.

Vahaboglu, H., Ozturk, R., Aygun, G., Coskunkan, F., Yaman, A., Kaygusuz, A., Leblebicioglu, H., Balik, I., Aydin, K. \& Otkun, M. (1997). Widespread detection of PER-1-type extended-spectrum $\beta$ lactamases among nosocomial Acinetobacter and Pseudomonas aeruginosa isolates in Turkey: a nationwide multicenter study. Antimicrob Agents Chemother 41, 2265-2269.

Weldhagen, G. F., Poirel, L. \& Nordmann, P. (2003). Ambler class A extended-spectrum $\beta$-lactamases in Pseudomonas aeruginosa: novel developments and clinical impacts. Antimicrob Agents Chemother 47, 2385-2392. 